\title{
Performance Evaluation of Signal Strength Based Handover Algorithms
}

\author{
Sanjay Dhar ROY \\ Affiliation1 National Institute of Technology, Durgapur, India \\ Email:s_harroy@yahoo.com \\ Received November 27, 2008; revised March 21, 2009; accepted June 7, 2009
}

\begin{abstract}
Performance evaluation of handover algorithms has been studied for mobile cellular network. Effects of averaging, hysteresis margin and shadow fading are investigated for different handoff algorithms. Probability of outage, handover delay and average number of handovers are considered as performance metrics. Different handover algorithms considered here are based on relative signal strength with hysteresis, relative signal strength with hysteresis and threshold, absolute signal strength and combined relative and absolute signal strength. Both analytical and simulation methods have been used in this paper. This study is important as performance analyses of cellular system, in presence of handoff, will be important for future generation wireless networks, for example, WiMAX, UMTS.
\end{abstract}

Keywords: Handoff, Algorithm, Averaging, Outage, Handoff Delay

\section{Introduction}

Signal strength at a Mobile Station (MS) depends upon path loss, shadow fading and multipath fading. Path loss depends on the distance of MS from a Base Transceiver Station (BTS). It increases with the distance from BTS. Between BTS and MS, there are many obstacles e.g., trees, buildings, vehicles. Those obstacles create variation of signal strength over the mean path loss. This variation is known as shadow fading which follows log normal distribution i.e. standard deviation of shadow fading $(\sigma)$ in $\mathrm{dB}$ follows normal or Gaussian distribution [3]. MS receives line of sight (LOS) signal from BTS and signals reflected from different places. Those multipath components result multipath fading. Multipath fading is found to follow Rayleigh distribution [1]. Signal averaging can filter out multipath fading. When MS moves from one BTS to another, on the way signal from current BTS get reduced whereas signal from other BTS increased. So, MS should be served by the new BTS when signal from serving BTS reduced below a specified level. This process of transferring control of MS from one BTS to another BTS without interruption of service is known as Handover. Handover or handoff is mainly of two types, hard handoff or soft handoff. Hard handoff is also referred to as "Break before Make connection". MS is connected to only one BTS at a time. Soft handoff refers to as "Make before Break connection". MS may be in connection with more than one BTS at a time. We have investigated performance evaluation for hard handoff case. Handover may also be classified as horizontal and vertical handover. Horizontal handoff takes place when MS moves from one cell to another cell of the same system e.g., GSM. Vertical handoff takes place when MS moves from one cell of a system to another cell of a different system e.g., GSM and WLAN. Handover process can be divided into mainly Initiation and Execution phase. In initiation phase based on some criteria viz., Received Signal Strength indicator (RSSI), BER, SIR, distance, velocity, it is checked if MS receives signal from BTS other than serving BTS then QoS will be better or not. Ideally, handover should depend on path loss and to some extent on shadow fading. To make handover decision independent of Raleigh fading, both uplink and downlink measurements are taken over a interval of 480 milliseconds time (sampling time) for averaging of fast fading effects in case of GSM. In practice, diversity techniques such as frequency hopping, antenna diversity and signal processing such as convolution coding, equalizers are used to handle Rayleigh fading. Long term shadow fading is compensated by increasing power budget margin increasing transmit power and co-channel reuse distance. If handover does not take place at right time then an ongoing call may be dropped. To prevent call drop before handoff due to unavailability of channel, several handoff prioritization scheme are proposed e.g., 
Guard channel scheme, Queuing of handoff [4]. In execution phase, once the need of handoff is detected, MS receives new channel in association with Base station controller (BSC) and Mobile switching center (MSC). Several Handover analyzes have been made so far $[1,2$, $5,9]$. Handoff in cellular systems was summarized in [4]. Description about macro cell, micro cell, corner effects were also provided in [4]. Vijayan et al. provides a framework considering level crossing analysis for performance evaluation of handoff algorithms [1]. Effects of correlation for shadow fading were investigated based on measurements [6]. A closed form expression for handoff rate was proposed in [8]. Handover initiation can be based on various approaches viz., relative signal strength, relative signal strength with threshold, relative signal strength with hysteresis, relative signal strength with hysteresis and threshold, prediction technique, distance, velocity, combined relative and absolute signal strength [7]. Our approach considers relative signal strength with hysteresis and absolute signal strength. Performance of handover algorithm can be determined based on criteria viz., number of unnecessary handoffs, probability of outage, average number of handoffs, handover delay, and probability of blocking [7]. We have analyzed the performance of the algorithm based on probability of outage, handover delay, and number of handovers. Effects of shadow fading, averaging interval, hysteresis margin (h) are considered on these parameters. Singh et al. [10] suggested that $\mathrm{h}=\mathrm{e} \times \sigma$ where $\mathrm{e}=1.3-1.6$ and over the coverage area, $\mathrm{h}$ must be dynamically adjusted as a function of $\sigma$. Number of handovers is traded off against handover delay (HO delay) in several papers $[1,9]$. Simulation study [2] is performed to find the effect of type and length of averaging window on handover performance. In all cases number of handovers should be small as it would reduce switching load of MSC.

Organization of this paper: In Section 2, system model is presented. Then probability of outage, $\mathrm{P}_{\text {out }}$ and probability of handover or assignment, $\mathrm{P}_{\text {assn }}$ are calculated using simple analytical model considering handover based on absolute signal strength measurements. Effect of shadow fading on $\mathrm{P}_{\text {out }}$ is also analyzed. Section 3 describes simulation model to obtain handover delay and number of handovers considering averaging of signal strength and other parameters. In Section 4, numerical results are presented. Finally in Section 5, conclusion is stated.

\section{System Model}

Two base stations, BTS1 and BTS2 are separated by D meters $[1,10]$. Mobile station (MS) is moving from BTS1 to BTS2 with constant speed. The signal level received from two BTSs (in $\mathrm{dB}$ ) at a distance, $\mathrm{d}$ from BTS1 can be expressed as follows:

$$
\begin{aligned}
& P_{r x 1}(d)=K_{1}-K_{2} \log _{10}(d)+x_{1}(d) \mathrm{d} €(0, \mathrm{D}) \text { meter. (1) } \\
& P_{r x 2}(d)=K_{1}-K_{2} \log _{10}(D-d)+x_{2}(d)
\end{aligned}
$$

$\mathrm{P}_{\mathrm{rx} 1}(\mathrm{~d})$ and $\mathrm{P}_{\mathrm{rx} 2}(\mathrm{~d})$ are received signal from BTS1 and BTS2 respectively at a distance d meters from BTS1. Rayleigh fading is neglected since it has shorter correlation distance compared to shadow fading. $\mathrm{K}_{1}$ and $\mathrm{K}_{2}$ are due to path losses. $\mathrm{K}_{2}$ is actually $10 \mathrm{n}$, where $\mathrm{n}$ is path loss component. We assume that $\mathrm{K}_{1}=0$ and $\mathrm{K}_{2}=30$. $\mathrm{x}_{1}(\mathrm{~d})$ and $\mathrm{x}_{2}(\mathrm{~d})$ are two independent zero mean stationary Gaussian processes. Hence received power from BTSs may also be considered to be Gaussian processes with mean, $\mu_{1}=K_{1}-K_{2} \log (d)$ and $\mu_{2}=K_{1}-K_{2} \log (D-d)$ respectively. $\mathrm{x}_{1}(\mathrm{~d})$ and $\mathrm{x}_{2}(\mathrm{~d})$ are assumed to have exponential correlation proposed by Gudmundson[6] based on experimental results. That is, $\mathrm{E}\left\{\mathrm{x}_{1}\left(\mathrm{~d}_{1}\right) \mathrm{x}_{1}\left(\mathrm{~d}_{2}\right)\right\}=\mathrm{E}\left\{\mathrm{x}_{2}\left(\mathrm{~d}_{1}\right)\right.$ $\left.\mathrm{x}_{2}\left(\mathrm{~d}_{2}\right)\right\}=\sigma^{2} \exp \left(-\mathrm{d}_{\mathrm{s}} / \mathrm{d}_{0}\right)$. Where $\mathrm{d}_{0}$ is correlation distance which determines the decaying factor for correlation.

\subsection{Handover Algorithm for Absolute Signal Strength Method}

When received signal from BTS1 is less than a specified value and at the same time received signal from BTS2 is more than minimum value of received signal for continuation of a call then handover (HO) will take place from BTS1 to BTS2. Similarly condition for handover from BTS2 to BTS1 can be stated as follows.

$$
\begin{aligned}
& \mathrm{P}_{\text {rx1 }}(\mathrm{d})<\mathrm{P}_{\text {rho }} \text { and } \mathrm{P}_{\text {rx2 }}(\mathrm{d})>\mathrm{P}_{\text {rmin }}: \text { HO: BTS } 1 \rightarrow \mathrm{BTS} 2 \\
& \mathrm{P}_{\mathrm{rx} 2}(\mathrm{~d})<\mathrm{P}_{\text {rho }} \text { and } \mathrm{P}_{\mathrm{rx} 1}(\mathrm{~d})>\mathrm{P}_{\text {rmin }}: \text { HO: BTS } 2 \rightarrow \mathrm{BTS} 1
\end{aligned}
$$

where $P_{\text {rho }}=$ Absolute value of received power from any BTS after which handover should take place. $\mathrm{P}_{\mathrm{rmin}}=$ Minimum value of received power for which call is possible. If signal strength becomes less than $\mathrm{P}_{\mathrm{rmin}}$ then there will be call drop for ongoing call and new call will not be possible.

At a distance, $d$ from BTS1 if received signal strengths from both BTSs go below $\mathrm{P}_{\text {rmin }}$ then call will not be possible i.e, there will be outage. Probability of outage,

$$
\begin{aligned}
& P_{\text {out }}=\operatorname{prob}\left(P_{r x 1}(d) \leq P_{r \text { min }} \text { and } P_{r \times 2}(d) \leq P_{r \text { min }}\right) \\
& P_{\text {out }}=\operatorname{Pr} o b\left(P_{r \times 1}(d) \leq P_{r \text { min }}\right) \text { and } \operatorname{Pr} o b\left(P_{r \times 2}(d) \leq P_{r \text { min }}\right)
\end{aligned}
$$

(Since these two events are statistically independent)

$$
P_{\text {out }}=Q\left(\frac{\left(\mu_{1}-P_{r \min }\right)}{\sigma}\right) \times Q\left(\frac{\left(\mu_{2}-P_{r \min }\right)}{\sigma}\right)
$$

$Q(x)$ is Q-function. $P(X \geq x)=Q(x)$ for $X \sim N(0,1)$

$$
\begin{aligned}
& \text { If } \quad X \sim N(\mu, \sigma) \quad \text { then } \quad P(X \geq x)=Q\left(\frac{(x-\mu)}{\sigma}\right) \quad \text { and } \\
& P(X \leq x)=Q\left(\frac{(\mu-x)}{\sigma}\right)=1-Q\left(\frac{(x-\mu)}{\sigma}\right)
\end{aligned}
$$

Mean of received powers are distance dependent. Us- 
ing a computer program, varying d, we have plotted Figure 1. Keeping distance fixed, varying $\sigma$, we have plotted Figure 2 and Figure 3. When received signal from serving BTS will be less than $\mathrm{P}_{\text {rho }}$ then there will be handover to other BTS. Current BTS should be able to serve the MS i.e., received power from it should be more than $\mathrm{P}_{\text {rmin }}$. So probability of assignment (or handover) to any BTS can be obtained as follows: Probability of assignment to BTS1,

$$
P_{a s s n 1}=\operatorname{prob}\left(P_{r x 1}(d) \leq P_{r h o} a n d P_{r x 2}(d) \geq P_{r \text { min }}\right)
$$

(Since these two events are statistically independent)

$$
P_{\text {assnl }}=Q\left(\frac{\left(\mu_{1}-P_{r h o}\right)}{\sigma}\right) \times Q\left(\frac{\left(P_{r \min }-\mu_{2}\right)}{\sigma}\right)
$$

Similarly, probability of assignment (or handover) to BTS2 can be obtained as follows:

$$
P_{\text {ass } 2}=Q\left(\frac{\left(\mu_{2}-P_{r h o}\right)}{\sigma}\right) \times Q\left(\frac{\left(P_{r \min }-\mu_{1}\right)}{\sigma}\right)
$$

Using the above equation we have plotted Figure 4. It is noticed that at 1000 meter Probability of handover is maximum, where MS can be assigned to any BTS. Shadow fading effect will be maximum there (Figure 3).

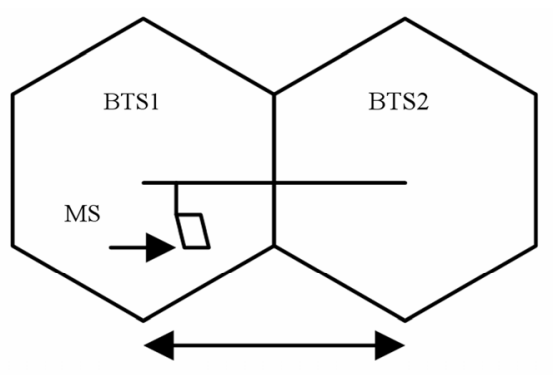

D meter

Figure 1. System model.

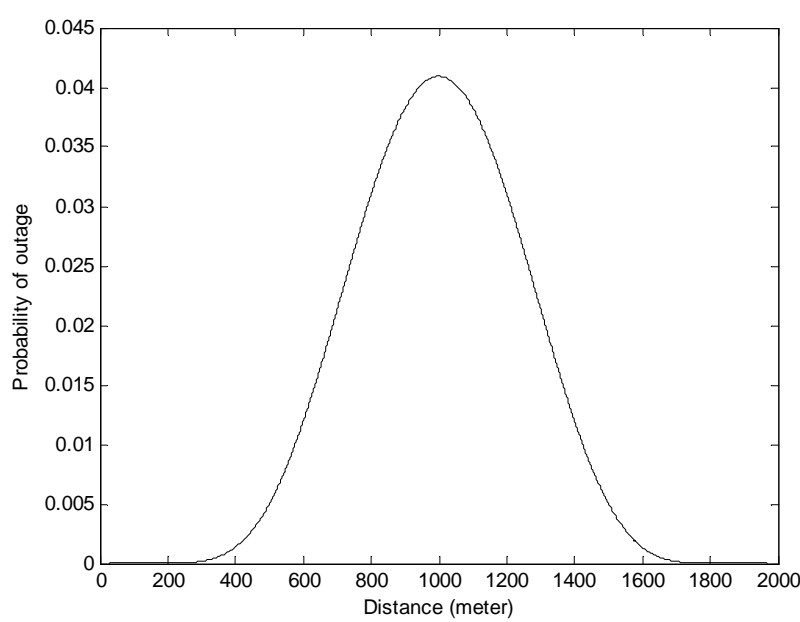

Figure 2. Probability of outage vs. distance.

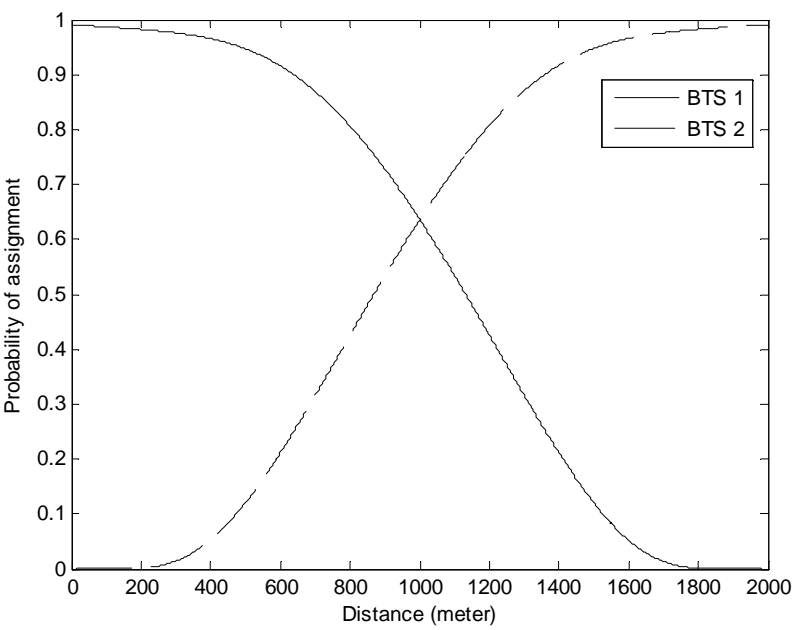

Figure 3. Probability of assignment to a BTS vs. distance.

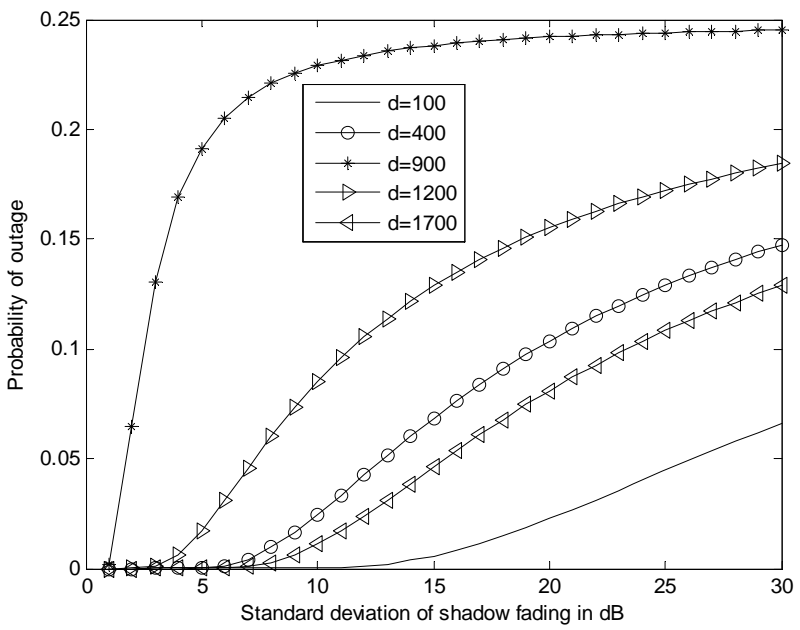

Figure 4. Probability of outage vs. standard deviation of shadow fading.

\section{Simulation Model}

Received signal strength is sampled at discrete time instants, $\mathrm{t}_{\mathrm{i}}=\mathrm{kt}_{\mathrm{s}}$. $\mathrm{t}_{\mathrm{s}}$ is sampling time. And corresponding sampling interval in distance is $\mathrm{d}_{\mathrm{s}}=\mathrm{vt}_{\mathrm{s}}$. Here $\mathrm{v}$ is constant velocity of the mobile. $t_{s}$ is $480 \mathrm{~ms}$ (nearly equal to $0.5 \mathrm{sec}$ ) in case of GSM. We assume $\mathrm{v}=2 \mathrm{~m} / \mathrm{sec}$ so that $\mathrm{d}_{\mathrm{s}}$ is 1 meter. Received signal strengths from both BTSs are averaged using exponential averaging window. Received signal strengths from BTSs sampled at $\mathrm{kd}_{\mathrm{s}}$ distance corresponding to $\mathrm{t}_{\mathrm{i}}$ are respectively as follows:

$$
\begin{aligned}
& P_{r x 1}\left(k d_{s}\right)=K_{1}-K_{2} \log _{10}\left(k d_{s}\right)+x_{1}\left(k d_{s}\right) \\
& P_{r x 2}\left(k d_{s}\right)=K_{1}-K_{2} \log _{10}\left(D-k d_{s}\right)+x_{2}\left(k d_{s}\right)
\end{aligned}
$$

These received signal strengths are averaged using discrete time counterpart of exponential averaging window [8] as shown below. To generate the shadow fading component, correlation of shadow fading is considered. 
Using recursive relations fading components have been generated and the same have been used for simulation purpose.

$$
\begin{gathered}
P_{r x 1, a v g}(k)=e^{-\left(\frac{d_{s}}{d_{a v}}\right)} P_{r x 1, a v g}(k-1)+\left(1-e^{-\left(\frac{d_{s}}{d_{a v}}\right)}\right) P_{r x 1}(k) \text { (8) } \\
P_{r x 2, a v g}(k)=e^{-\left(\frac{d_{s}}{d_{a v}}\right)} P_{r x 2, a v g}(k-1)+\left(1-e^{-\left(\frac{d_{s}}{d_{a v}}\right)}\right) P_{r x 2}(k) \text { (9) }
\end{gathered}
$$

where $d_{a v}$ is length of averaging window and $h$ is hysteresis margin. $\mathrm{P}_{\mathrm{rx} 1, \mathrm{avg}}(\mathrm{k})$ is the averaged received signal from BTS1 at $\mathrm{kd}_{\mathrm{s}}$ distance. $\mathrm{P}_{\mathrm{r} \times 1, \text { avg }}(\mathrm{k}-1)$ is the averaged received signal from $B T S 1$ at $(k-1) d_{s}$ distance. $P_{r \times 2, a v g}(k)$ is the averaged received signal from $\mathrm{BTS} 2$ at $\mathrm{kd}_{\mathrm{s}}$ distance.

\subsection{Handover Algorithm for Relative Signal Strength with Hysteresis}

If received signal from BTS1 is less than received signal from BTS2 by a margin, $h$ then handover will be there from BTS1 to BTS2. Similarly, if received signal from BTS2 is less than received signal from BTS1 by a margin, $\mathrm{h}$ then handover will be there from BTS2 to BTS1. We can express these using following simple relations.

$$
\begin{array}{ll}
{\left[\mathrm{P}_{\mathrm{rx} 2 \text {,avg }}(\mathrm{k})-\mathrm{P}_{\mathrm{rx} 1 \text {,avg }}(\mathrm{k})\right]>+\mathrm{h}} & \text { HO: BTS1 } \rightarrow \text { BTS2 } \\
{\left[\mathrm{P}_{\mathrm{rx} 2 \text {,avg }}(\mathrm{k})-\mathrm{P}_{\mathrm{rx} 1, \mathrm{avg}}(\mathrm{k})\right]<-\mathrm{h}} & \text { HO: BTS2 } \rightarrow \text { BTS1 }
\end{array}
$$

\subsection{Handover Algorithm for Relative Signal Strength with Threshold}

If received signal from BTS1 is less than received signal from BTS2 by a margin, $h$ and received signal from BTS2 is greater than a threshold value then handover will be there from BTS1 to BTS2. Similarly, if received signal from BTS2 is less than received signal from BTS1 by a margin, $\mathrm{h}$ and received signal from BTS 1 is greater than a threshold value then handover will be there from BTS2 to BTS1. We can express these using following simple relations. For simplicity, we consider threshold value equal to $\mathrm{P}_{\text {rmin }}$.

$$
\begin{aligned}
& {\left[\mathrm{P}_{\mathrm{rx} 2, \mathrm{avg}}(\mathrm{k})-\mathrm{P}_{\mathrm{rx} 1, \mathrm{avg}}(\mathrm{k})\right]>+\mathrm{h} \text { and }\left[\mathrm{P}_{\mathrm{rx} 2, \mathrm{avg}}(\mathrm{k})>\mathrm{P}_{\mathrm{rmin}}\right]} \\
& \text { HO: BTS1 } \rightarrow \text { BTS } 2 \\
& {\left[\mathrm{P}_{\mathrm{rx} 1, \mathrm{avg}}(\mathrm{k})-\mathrm{P}_{\mathrm{rx} 2, \mathrm{avg}}(\mathrm{k})\right]>+\mathrm{h} \text { and }\left[\mathrm{P}_{\mathrm{rx} 1, \mathrm{avg}}(\mathrm{k})>\mathrm{P}_{\mathrm{rmin}}\right]} \\
& \text { HO: BTS2 } \rightarrow \text { BTS } 1
\end{aligned}
$$

\subsection{Handover Algorithm for Combined Relative and Absolute Signal Strength Method}

If received signal from BTS1 is less than received signal BTS2 by a margin, $h$ and received signal from BTS1 is less than a threshold value $\left(\mathrm{P}_{\text {rho }}\right)$ then handover will be there from BTS1 to BTS2. Similarly, if received signal from BTS2 is less than received signal BTS1 by a margin, $h$ and received signal from BTS2 is less than a threshold value then handover will be there from BTS2 to BTS1. We can express these using the following simple relations.

$$
\begin{aligned}
& {\left[\mathrm{P}_{\mathrm{rx} 2 \text {,avg }}(\mathrm{d})-\mathrm{P}_{\mathrm{rx} 1 \text {,avg }}(\mathrm{d})\right]>+\mathrm{h} \text { and }\left[\mathrm{P}_{\mathrm{rx} 1 \text {,avg }}(\mathrm{d})<\mathrm{P}_{\text {rho }}\right] \text { : }} \\
& \text { HO: BTS1 } \rightarrow \text { BTS2 } \\
& {\left[\mathrm{P}_{\mathrm{rx} 1 \text {,avg }}(\mathrm{d})-\mathrm{P}_{\mathrm{rx} 2 \text {,avg }}(\mathrm{d})\right]>+\mathrm{h} \text { and }\left[\mathrm{P}_{\mathrm{rx} 2 \text {,avg }}(\mathrm{d})<\mathrm{P}_{\mathrm{rho}}\right] \text { : }} \\
& \text { HO: BTS2 } \rightarrow \text { BTS } 1
\end{aligned}
$$

Using these algorithms after large number of iterations average number of handovers $\left(\mathrm{N}_{\mathrm{ho}}\right)$, handover delays are calculated and plotted against hysteresis margin, standard deviation of shadow fading component.

\section{Numerical Results}

Following values are chosen for the analysis purpose:

1) Standard deviation of shadow fading, $\sigma=6 \mathrm{~dB}$

2) Distance between BTSs, D = 2000 meter.

3) Correlation distance, $d_{0}=20$ meter.

4) Length of averaginvg window, $d_{a v}=10$ meter and 20 meter.

5) Velocity of mobile station, $v=2$ meter $/ \mathrm{sec}$.

6) Sampling time, $t_{\mathrm{s}}=0.5 \mathrm{sec}$

7) Sampling distance, $d_{s}=v t_{s}=1$ meter.

8) $\mathrm{P}_{\text {rmin }}=-95 \mathrm{~dB} 9 . \mathrm{P}_{\text {rho }}=-85 \mathrm{~dB}$

Analytical results are shown in Figure 2 to Figure 4. In Figure 2, $\mathrm{P}_{\text {out }}$ is plotted against distance from BTS1. $\mathrm{P}_{\text {out }}$ is large near the boundary of the cells and it is zero near to any of the BTSs. Figure 3 illustrates where handoff will take place and corresponding assignment probability is shown in this figure.

Figure 4 shows effects of shadow fading on probability of outage are shown. Figure 4 considers $d=100,400$, 1700 i.e, very near to either of BTSs. Naturally probability of outage is very less, almost zero for small $\sigma$, but for large values of $\sigma$, outage is possible for the specified values. Figure 4 also considers distance near the cell boundary $(d=900,1200)$ where the signal from either of BTSs is very low, so we see that $\mathrm{P}_{\text {out }}$ largely depends on $\sigma$. Near to the cell boundary, probability is very large.

Figure 5 to Figure 14 shows simulation results. Average number of handoffs and handover delay are plotted against $h$ for different values of $d_{a v}$. We consider delay or handover delay to be the distance where first handover occurs. Actually, handover delay is total of averaging delay and hysteresis delay. Hysteresis time: it is the time needed when MS moves some distance away after measurements. It can be noticed from the figures Figure 5 to Figure 7 that handover delay increases with increasing h. And handover delay increases when averaging distance 


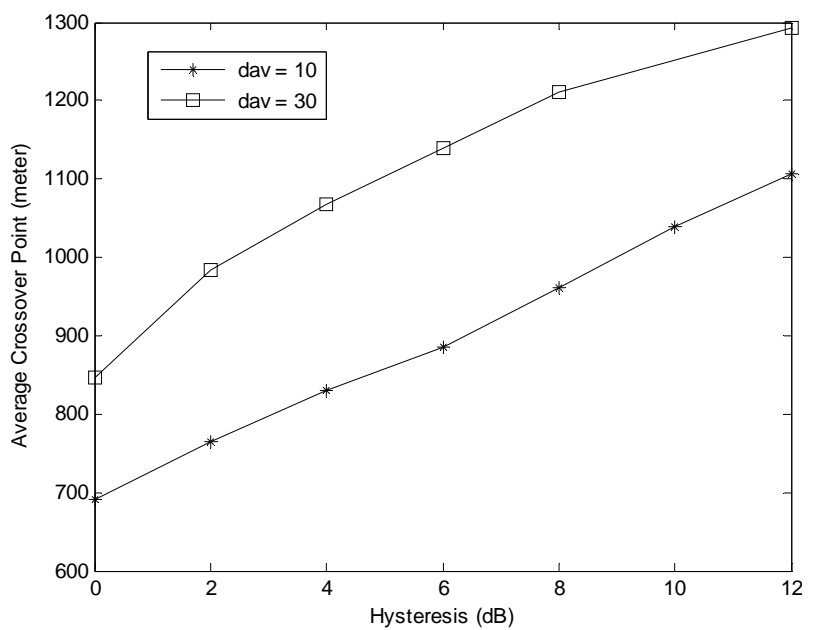

Figure 5. Handover delay vs. hysteresis margin for relative signal strength with hysteresis.

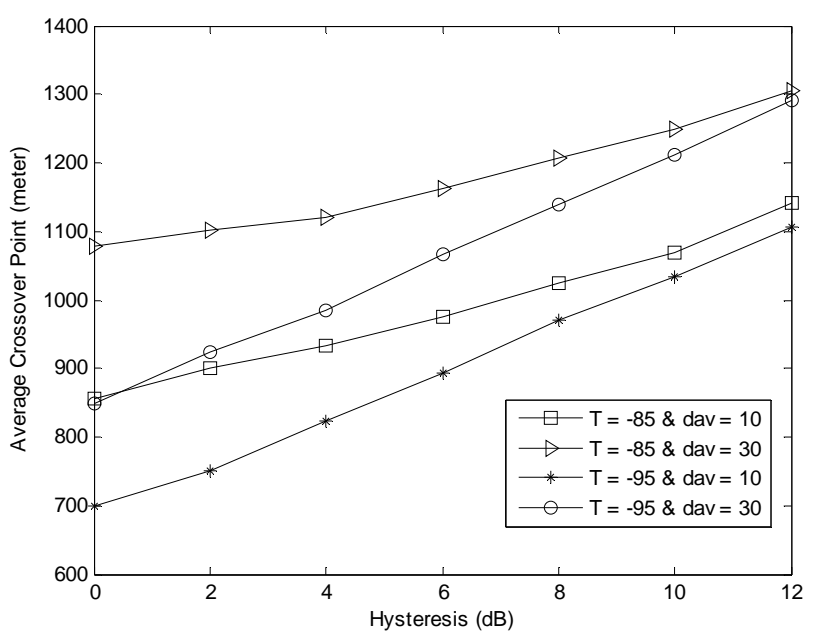

Figure 6. Handover delay vs. hysteresis margin for relative signal strength with threshold and hysteresis.

is increased. Since more averaging consumes more time. There is no significant change in handover delay (with $\mathrm{d}_{\mathrm{av}}=30$ ) with respect to $\sigma$ after considering its correlation. That is due to averaging of signal strength. Averaging of signals filter out multipath component and to some extent shadow fading variation. For this reason delay vs. $\sigma$ plot is not shown. Figure 5 shows variation of handoff delay for relative RSS for $\mathrm{d}_{\mathrm{av}}=10$ and 30 . Handoff occurs near to cell boundary for $\mathrm{d}_{\mathrm{av}}=10$ and $\mathrm{h}=12$. Hystersis value is to be very large for avoiding ping-pong for this algorithm. Figure 6 shows variation of crossover point for relative signal strength with hystersis and threshold. Four curves have been plotted for different threshold and $\mathrm{d}_{\mathrm{av}}$ values. Handover delay will not change with $\mathrm{T}$ for large values of $\mathrm{h}$ and $\mathrm{d}_{\mathrm{av}}$. For example, at $\mathrm{h}$ $=12$ and $\mathrm{d}_{\mathrm{av}}=30$, handoff delays for $\mathrm{T}=-85$ and -95 are same.

Figure 7 shows handover delay for combined relative and absolute signal strength based algorithm (CSS). Handover delay is more for $\mathrm{T}=-95 \mathrm{~dB}$ as this allows MS to be connected to the serving BTS for long compared to that with $\mathrm{T}=-85 \mathrm{~dB}$. Crossover point is more with $\mathrm{d}_{\mathrm{av}}=$ 30 than with $\mathrm{d}_{\mathrm{av}}=10$ meter because of large averaging.

It is observed that numbers of handoffs are less for large values of hysteresis. For $\mathrm{d}_{\mathrm{av}}=30$, number of handoffs is almost equal to one i.e, no unnecessary handovers for the specified values. For $\mathrm{d}_{\mathrm{av}}=10$, number of handoffs is large. That means less averaging may lead to unnecessary handoffs. Figure 8 shows number of handoff vs. hysteresis for relative signal strength basesd algorithm. Number of handoff is less for $\mathrm{d}_{\mathrm{av}}=30$ meter due to large averaging window length. Figure 9 shows variation of $\mathrm{N}_{\text {ho }}$ with $\mathrm{h}$ for CSS algorithm for different values of $\mathrm{T}$ and $d_{a v}$. Number of handoff is lowest for $T=-95 d B$ and $\mathrm{d}_{\mathrm{av}}=30$. This happens because the current BTS keeps control of MS for longer time. Figure 10 shows variation

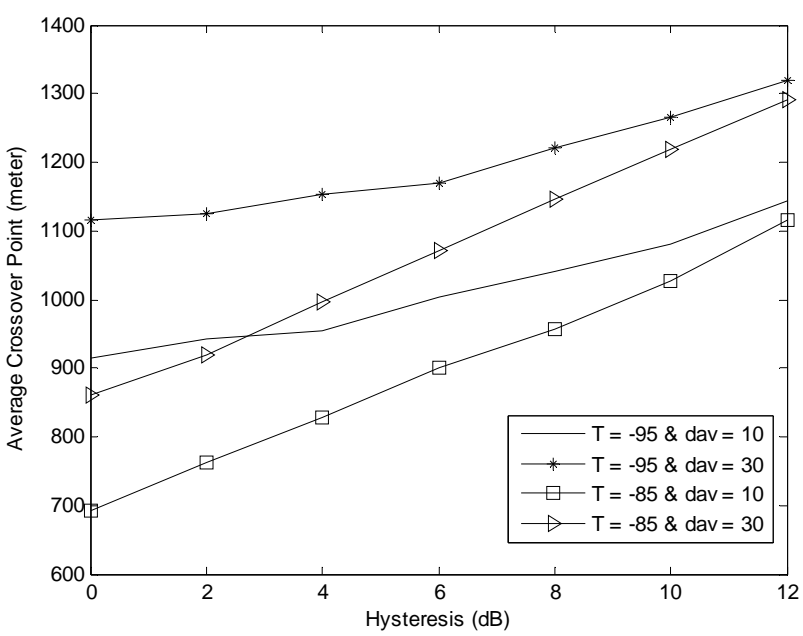

Figure 7. Handover delay vs. hysteresis margin for combined relative \& absolute signal strength with hysteresis method.

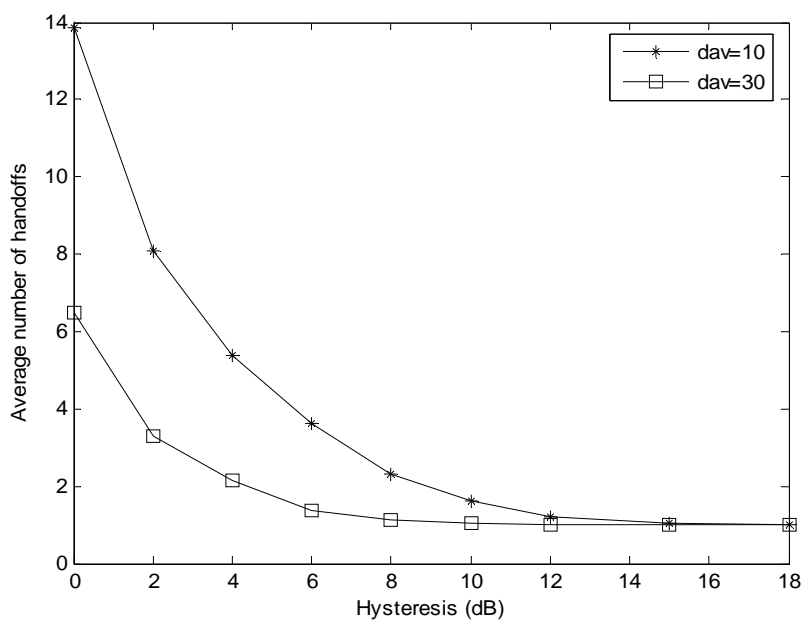

Figure 8. Average number of handoff vs. hysteresis margin for relative RSS. 
of $\mathrm{N}_{\text {ho }}$ with $\mathrm{h}$ for relative signal strength with threshold and hysteresis based algorithm for different values of $\mathrm{T}$ and $\mathrm{d}_{\mathrm{av}}$. Number of handoff is lowest for $\mathrm{T}=-85 \mathrm{~dB}$ and $\mathrm{d}_{\mathrm{av}}=30$. This happens because the current BTS keeps control of MS for longer time and control is transferred to candidate BTS only after it provides very good signal strength to sustain good quality and avoid ping pong. We have analyzed three different handover algorithms. Results suggest that effect of $\sigma, h$ and $d_{a v}$ similar for all different algorithms.

Next three figures show tradeoff curves for all three different handoff algorithms considered in this paper. Fig 11 show tradeoff for relative signal strength based handoff algorithm. Tradeoff curve provides an idea for choosing handover design parameter. Handoff parameter may be chosen for point where $\mathrm{N}_{\text {ho }}$ and Cross over point both are low. Figure 12 shows tradeoff for CSS algorithm

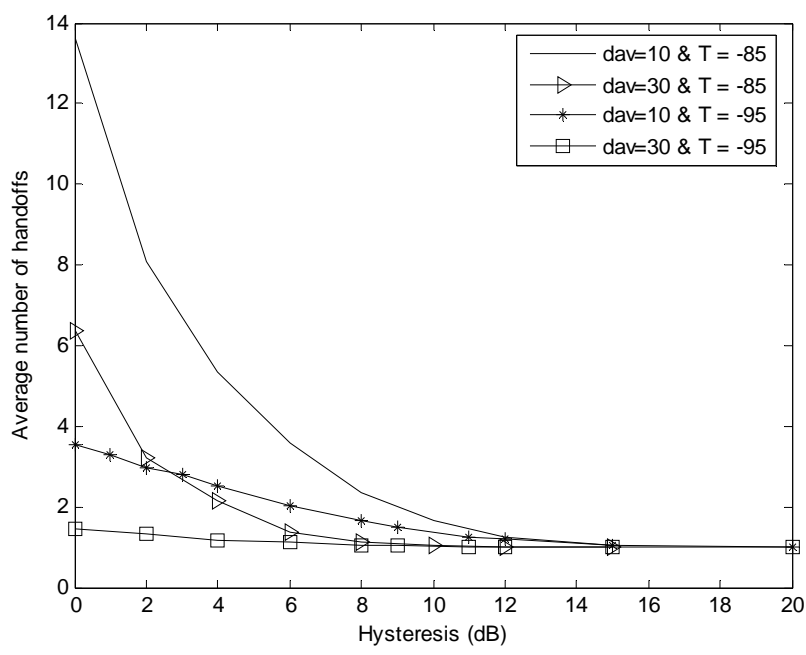

Figure 9. Average number of handoff vs. hysteresis margin for CSS.

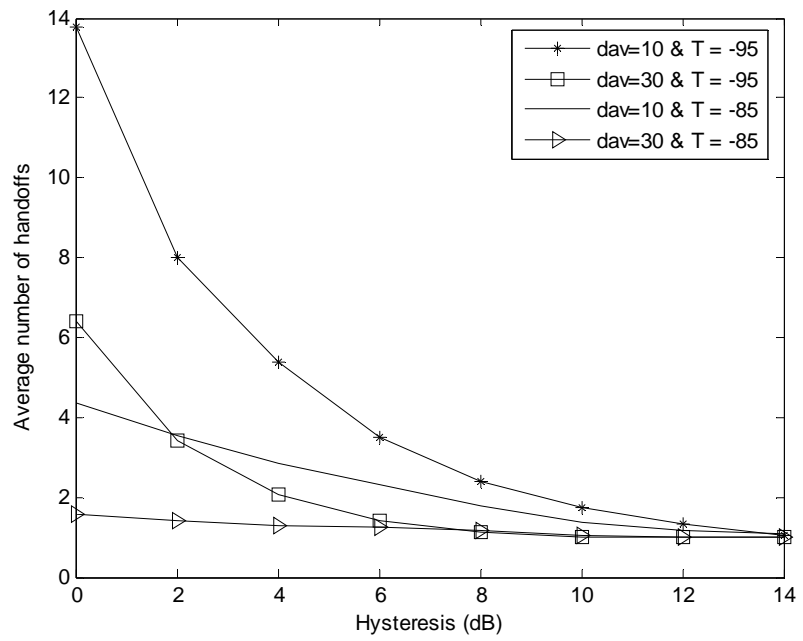

Figure 10. Average number of handoff vs. hysteresis margin for relative signal strength with threshold and hysteresis.

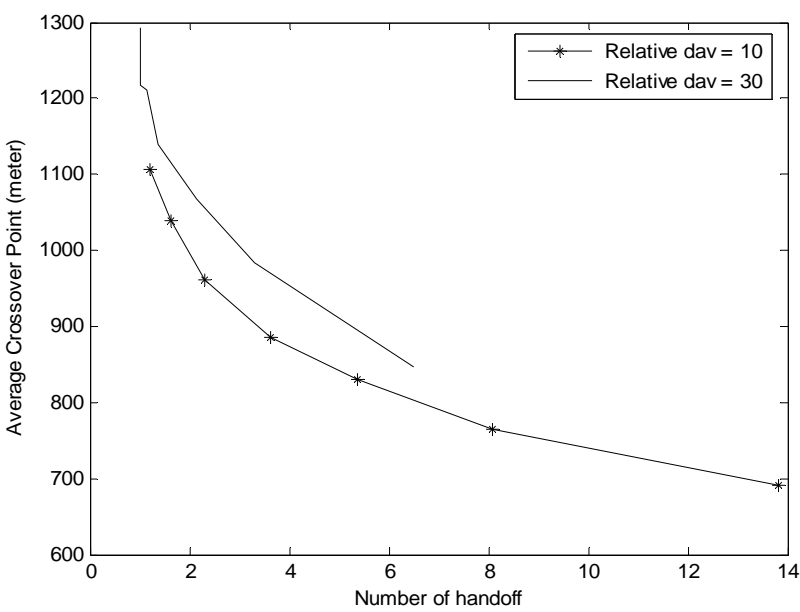

Figure 11. Tradeoff for relative RSS based algorithm.

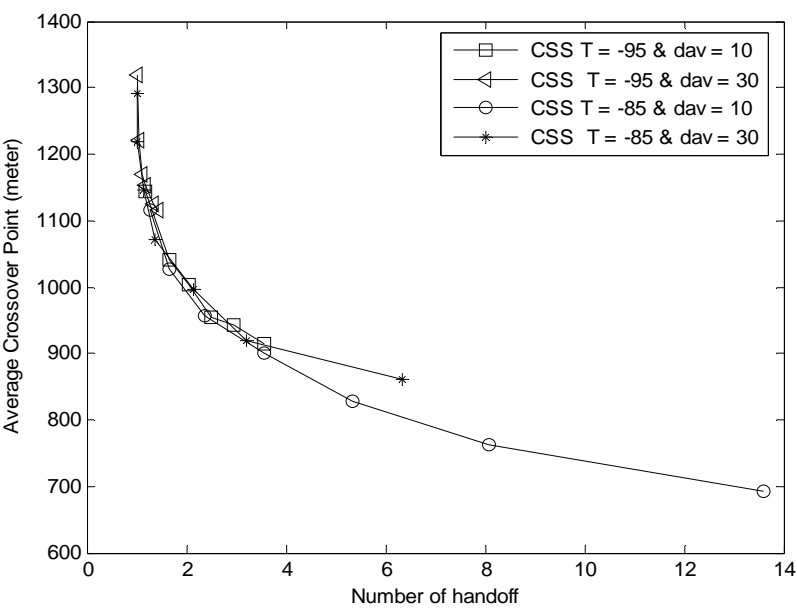

Figure 12. Tradeoff for relative CSS based algorithm.

for different values of $d_{a v}$ and $T$. $N_{h o}$ is one when cross over point is almost 1100 meter. Hence CSS algorithm provides very good balance between two conflicting parameters $\mathrm{N}_{\text {ho }}$ and handoff delay. Figure 12 shows tradeoff for relative signal strength with threshold and hysteresis based algorithm for different values of $d_{a v}$ and $T$. $\mathrm{N}_{\mathrm{ho}}$ is one when cross over point is almost 1200 meter. Finally, Figure 14 shows tradeoff curve for all three different algorithms. Two algorithms other than relative signal strength based handoff algorithm, provides almost same performance with proper choice of hysteresis value. Crossover point can be around 1200 meters with proper setting of hystersis value for $\mathrm{N}_{\mathrm{ho}}=1$ (one).

\section{Conclusions}

This paper presents very simple method to choose handover design parameters (e.g., averaging window length, hysteresis margin, standard deviation of shadow fading) for Mobile Cellular system. It uses analytical method for finding probability of outage and it uses simulation 
method for finding handover delay and average number of handoffs. Analysis and simulation results are obtained for three different algorithms. Absolute signal strength based algorithm has to be considered for intersystem handoff, as relative measurements are not possible for different cellular systems because of their different power requirement and other criteria. If candidate signal strength is not large enough then there may be ping-pong effect. Relative signal strength with hysteresis and threshold takes care of this. If serving BS strength is enough to provide good quality of service then a handoff to candidate BS may be considered as unnecessary. Hence, a combined absolute and relative signal strength based handoff algorithm can take care of this problem. Both of these two algorithms prevent unnecessary handoff by increasing handoff delay to some extent. Handoff decision criteria can be critical when cell splitted (microcellular) to increase capacity and decrease power requirements of MS. When there is very small hysteresis

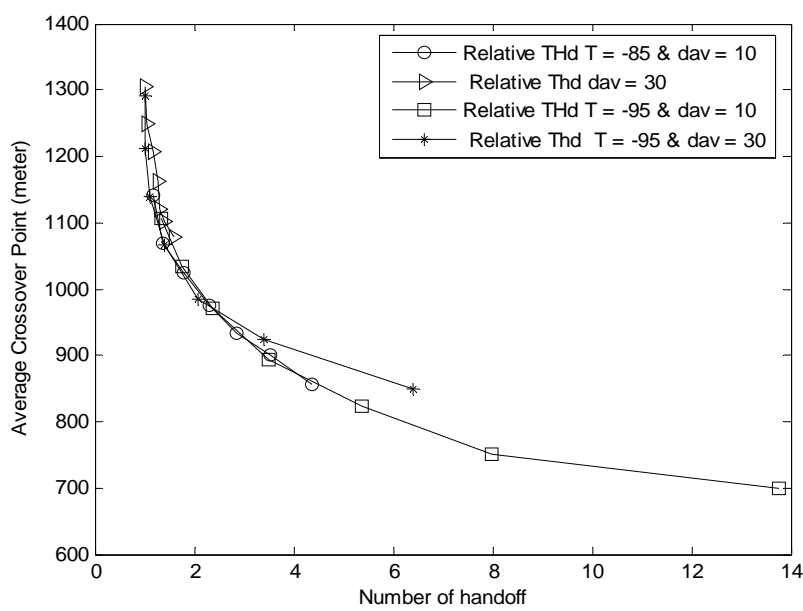

Figure 13. Tradeoff for relative RSS with threshold based algorithm.

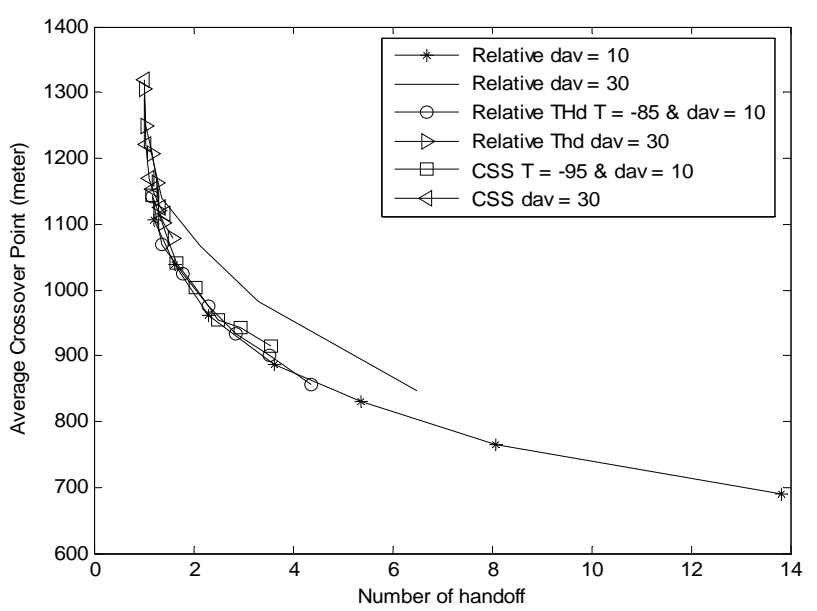

Figure 14. Tradeoff for all three algorithms together. margin or no hysteresis there may be ping-pong effect. Due to dynamic behaviour of propagation environment MS very close to BTS may be in deep fade for very short duration (e.g., street corner effect). Handover should not occur in such cases. To avoid this, averaging of signal strength may be done over short duration while keeping large hysteresis margin. Overlay macro cell may also be employed to overcome this problem. For microcellular systems short averaging time and large hystersis margin is more reliable and reverse for macro cellular systems. Probability of outage increases with increase in shadow fading. Since designer has almost no control over the shadow fading component, hysteresis margin can be dynamically varied to compensate the effect of shadow fading.

\section{Acknowledgments}

The author would like to thank Mr. A. Chandra, Dr. S. Kundu and Dr. S. K. Datta of NIT, Durgapur, India for their valuable suggestions.

\section{References}

[1] R. Vijayan and J. Holtzman, "A model for analyzing handoff algorithms," IEEE Transactions on Vehicular Technology, Vol. 42, No. 3, pp. 351-356, August 1993.

[2] G. E. Corazza, et al., "Characterization of handover initialization in cellular mobile radio networks," IEEE VTC' 94, pp. 1869-1872, 1994.

[3] T. S. Rappaport, "Wireless communications," Prentice Hall, 1996.

[4] D. Nisith, et al., "Handoff in cellular systems," IEEE Personal Communications, Dec. 1998.

[5] M. Gudmundson, "Analysis of Handover algorithm", IEEE VTC, May 1991, pp 537-42

[6] M. Gudmundson, "Correlation model for shadow fading in mobile radio systems," Electronics Letters, Vol. 27, No. 23, pp. 2145-2146, November 1991.

[7] G. P. Pollini, "Trends in handover design," IEEE Communication Magazine, Vol. 34, pp. 82-90, March 1996.

[8] G. P. Pollini, "Handover rate in cellular systems: Towards a closed form approximation," Global Telecommunications Conference, GLOBE COM'97, IEEE, Vol. 1, pp. 711-715, November 1997.

[9] N. Zhang and J. M. Holtzman, "Analysis of handover algorithms using both absolute and relative measurements," IEEE VTC'94, pp. 82-86, 1994.

[10] B. Singh, et al., "Sensitivity analysis of handover performance to shadow fading in microcellular systems," IEEE ICPWC, 2005.

[11] S. D. Roy, "Effects of averaging, shadow fading and hysteresis margin on handover performance," CD proceedings, ICEMC, Pesit, Bangalore, Aug. 2007. 